\title{
Output Feedback Control of Linear Systems with Input and Output Quantization
}

\author{
Daniel Coutinho
}

Minyue Fu

\author{
Carlos E. de Souza
}

\begin{abstract}
A considerable amount of research has been done on the use of logarithmic quantizers for networked feedback control systems. However, most results are developed for the case of a single quantizer (either measurement or control signal quantization). In this paper, we investigate the case of simultaneous input and output quantization for SISO linear output feedback systems. Firstly, we show that the problem of quadratic stabilization via quantized feedback can be addressed with no conservativeness by means of the sector bound approach. Secondly, we provide a bound on the maximal admissible sector bound via a scaled $\mathrm{H}_{\infty}$ optimization problem.
\end{abstract}

\section{INTRODUCTION}

The study of quantization errors in digital control systems has been an important area of research, since digital controllers were employed in feedback systems. Early works on quantized feedback concentrated on analyzing the effects of quantization and ways to mitigate them [1], [2]. The simplest approach to analyze the effects of quantized feedback control is to model the quantizer as sector bounded time-varying uncertainties and apply absolute stability theory tools.

Nowadays, many control systems are remotely implemented via communication channels with limited bandwidth which we will refer to as networked control systems. In such systems, the communication link is shared by different applications and a natural issue is to minimize the quantity of information needed to be transmitted while achieving a certain closed-loop performance. In the last several years, many researchers have concentred on this topic; see [3][8]. From the results proposed in [6], a new line of research focuses on the quadratic stabilization problem of linear timeinvariant (LTI) systems via quantized feedback [8], [9], which is referred to as the sector bound approach. In this methodology, the quantizer is assumed to be logarithmic, static and memoryless with fixed quantization levels. One can cite several advantages in employing logarithmic quantizers such as the ease on addressing the quadratic stabilization problem, explicit coarsest quantization density formulae, and the nice feature of needing only a few bits (in the context

This work was supported by CNPq, Brazil, under grants 45.4495/2007-2, 30.2741/05-4/PQ and 30.2317/02-3/PQ, and by ARC Centre for Complex Dynamic Systems and Control, Australia.

D. Coutinho is with the Group of Automation and Control Systems, Faculty of Engineering, Pontifícia Universidade Católica do Rio Grande do Sul, Av. Ipiranga 6681, Porto Alegre 90619-900, Brazil (dcoutinho@pucrs.br)

$\mathrm{M}$. Fu is with the School of Electrical Engineering and Computer Science, University of Newcastle, Callaghan, NSW 2308, Australia (minyue. fulnewcastle.edu.au)

C.E. de Souza is with the Department of Systems and Control, Lab. Nacional de Computação Científica, Petrópolis, RJ 25651-075, Brazil (csouza@lncc.br) of finite level quantization) to approximately achieve the performance of non-quantized feedback systems.

In networked control systems, the information (control signal and measurements) is generally exchanged through a shared communication channel among control system components (sensors, controller, actuator, etc.), thus we may logically suppose that both control and measurement signals are quantized [10]. However, up to now, very few results have addressed stability and stabilization problems for input and output quantized feedback systems with the exception of [10] and [11].

In this paper, we extend the sector bound approach [8] to cope with input and output quantization for single-input single-output (SISO) linear time-invariant output feedback systems. We show that the problem of quadratic stabilization via quantized feedback can be addressed with no conservatism by means of the sector bound approach. This result converts the quantized feedback control problem into a robust control problem. Moreover, we provide a bound on admissible quantization densities by introducing a scaling parameter on the equivalent robust condition. In the context of practical quadratic stability with finite level quantized feedback, we introduce a method for allocating the bandwidth of the communication channel, which is illustrated via a numerical example.

\section{Problem Formulation}

Consider the quantized feedback system in Figure 1. The system to be controlled is modeled by

$$
\left\{\begin{aligned}
x(k+1) & =A x(k)+B u(k) \\
y(k) & =C x(k)
\end{aligned}\right.
$$

where $A \in \mathbb{R}^{n \times n}, B \in \mathbb{R}^{n}, C \in \mathbb{R}^{1 \times n}, x$ is the state, $u$ is the control signal and $y$ is the measurement, and the dynamic controller is given by

$$
\left\{\begin{aligned}
\xi(k+1) & =A_{c} \xi(k)+B_{c} v(k) \\
w(k) & =C_{c} \xi(k)+D_{c} v(k)
\end{aligned}\right.
$$

The input and output quantizers are modeled by

$$
\left\{\begin{array}{l}
v(k)=Q_{1}(y(k)) \\
u(k)=Q_{2}(w(k))
\end{array}\right.
$$

where $Q_{1}(\cdot)$ and $Q_{2}(\cdot)$ are logarithmic quantizers with quantization densities $\rho_{1}$ and $\rho_{2}$, respectively. Without loss of generality, we assume that $(A, B, C)$ is a minimal realization of system (1) having the following transfer function

$$
G(z)=C(z I-A)^{-1} B \text {. }
$$




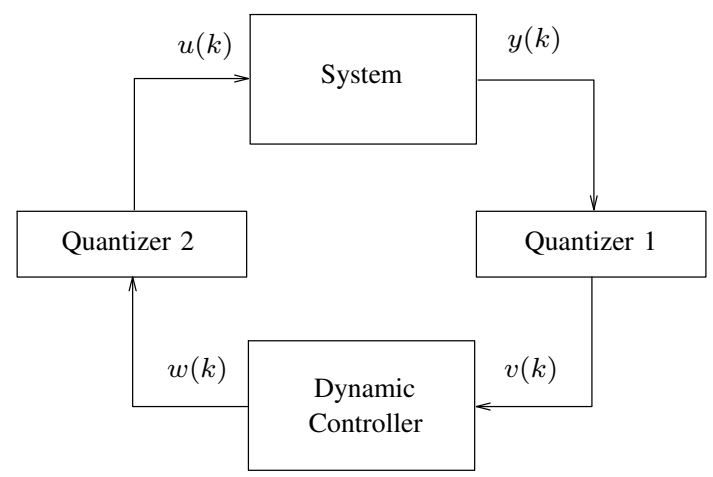

Fig. 1. Feedback Control with Input and Output Quantization by

A logarithmic quantizer $Q(\cdot)$ has quantization levels given

$$
\mathcal{V}=\left\{ \pm \mu_{i}: \mu_{i}=\rho^{i} \mu_{0}, i=0, \pm 1, \pm 2, \cdots\right\} \cup\{0\}, \mu_{0}>0
$$

where $\rho \in(0,1)$ represents the quantization density. A small $\rho$ implies coarse quantization, and a large $\rho$ means dense quantization. The quantizer $Q(\cdot)$ is depicted in Fig. 2 and is defined as follows:

$$
Q(\varepsilon)= \begin{cases}\rho^{i} \mu_{0}, & \text { if } \quad \frac{1}{1+\delta} \rho^{i} \mu_{0}<\varepsilon \leq \frac{1}{1-\delta} \rho^{i} \mu_{0}, \\ 0, & \text { if } \varepsilon=0 \\ -Q(-\varepsilon), & \text { if } \varepsilon<0\end{cases}
$$

where

$$
\delta=(1-\rho) /(1+\rho)
$$

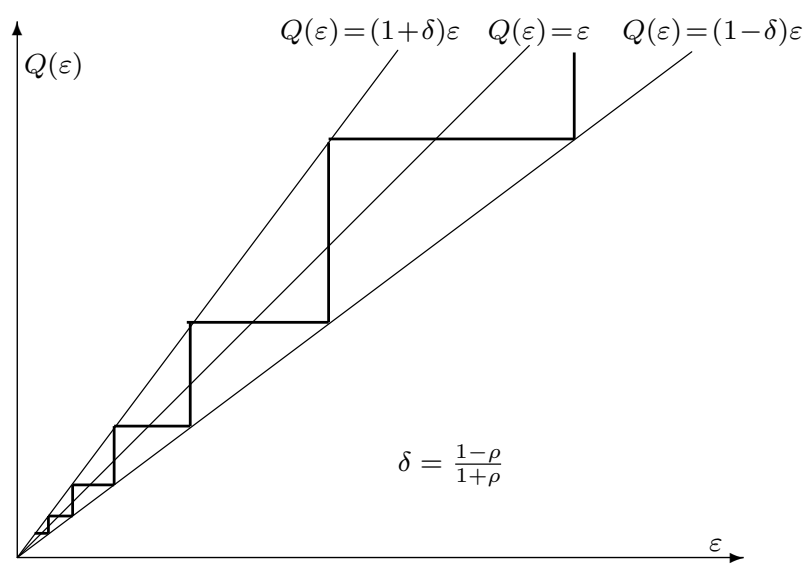

Fig. 2. Logarithmic Quantizer

The closed-loop system can be written as

$$
\left\{\begin{array}{l}
x(k+1)=A x(k)+B Q_{2}\left(C_{c} \xi(k)+D_{c} Q_{1}(C x(k))\right. \\
\xi(k+1)=A_{c} \xi(k)+B_{c} Q_{1}(C x(k))
\end{array}\right.
$$

which can be shortened as

$$
\bar{x}(k+1)=f\left(x(k), \xi(k), Q_{1}, Q_{2}\right)
$$

with $\bar{x}=\left[\begin{array}{ll}x^{T} & \xi^{T}\end{array}\right]^{T}$ and

$$
f\left(x, \xi, Q_{1}, Q_{2}\right)=\left[\begin{array}{c}
A x+B Q_{2}\left(C_{c} \xi+D_{c} Q_{1}(C x)\right) \\
A_{c} \xi+B_{c} Q_{1}(C x)
\end{array}\right] .
$$

In this paper, we assume that the input and output quantizers are independent with possibly different quantization densities, which is a natural setting in networked control systems. Under these conditions, we address the quadratic stabilization problem of the quantized closed-loop feedback system in (9). Further, in the finite quantization setup, we study the bandwidth allocation problem in the sense that quantization densities $\rho_{i}$ of the quantizers $Q_{i}(\cdot), i=1,2$, are chosen to minimize the communication channel bandwidth in some way.

\section{Previous Results}

In this section, we review some key results proposed in [8] where the quadratic stabilization problem of SISO linear feedback systems with a single quantizer is solved through sector bound technique and $H_{\infty}$ optimization.

Notice from Figure 2 that a logarithmic quantizer $Q(\varepsilon)$ can be bounded by a sector $(1+\Delta) \varepsilon$, where $\Delta \in[-\delta, \delta]$ and consider two possible configurations involving the system (1), controller (2) and a quantizer:

- Configuration I: the measurement is quantized, i.e. $v(k)=Q_{1}(y(k))$, but the control signal is not, i.e. $u(k)=w(k)$; and

- Configuration II: the control signal is quantized, i.e. $u(k)=Q_{2}(w(k))$, but the measurement is not, i.e. $v(k)=y(k)$.

If we consider the same LTI controller in (2) to either Configuration I or II, we extend from [8] the following result.

Theorem 3.1: Consider the system (1) and a single quantizer in Configuration I or II. For a given quantizer density $\rho$, this system is quadratically stabilizable via the controller (2) if and only if the auxiliary system

$$
\begin{aligned}
x(k+1) & =A x(k)+B w(k) \\
v(k) & =(1+\Delta) C x(k), \quad|\Delta| \leq \delta
\end{aligned}
$$

for Configuration I, or

$$
\begin{aligned}
x(k+1) & =A x(k)+B(1+\Delta) w(k) \\
y(k) & =C x(k), \quad|\Delta| \leq \delta
\end{aligned}
$$

for Configuration II, is quadratically stabilizable via the controller (2), where $\delta$ and $\rho$ are related by (7).

For both configurations, the supremum $\delta_{\text {sup }}$ of the sector bound $\delta$ for quadratic stabilization, which gives the smallest quantization density $\rho_{\text {inf }}$, is given by

$$
\delta_{\text {sup }}=\frac{1}{\inf _{A_{c}, B_{c}, C_{c}, D_{c}}\|\bar{G}(z)\|_{\infty}}
$$

where

$$
\bar{G}(z)=\frac{G(z) H(z)}{1-G(z) H(z)}, \quad H(z)=C_{c}\left(z I-A_{c}\right)^{-1} B_{c}+D_{c} .
$$


Proof. The proof of the equivalence between the quadratic stability of the quantized and the uncertain system can be found in [8, Theorem 3.2]. The result on $\delta_{\text {sup }}$ follows by noting that in both configurations the closed-loop system can be written as an open-loop transfer function $G(z) H(z)$ and a feedback loop $(1+\Delta)$, since $G(z) H(z)=H(z) G(z)$. The solution to $\delta_{\text {sup }}$ follows from the equivalence between quadratic stability and $H_{\infty}$ optimization [12], [13].

\section{MAIN RESUlts}

In this section, we extend the results of Theorem 3.1 to the double quantizer stabilization problem. Firstly, we show for quadratic stability analysis that input and output quantizers can be tackled with no conservatism by two sector bound conditions. Secondly, a sufficient control design condition is derived in terms of an $H_{\infty}$ optimization problem such that the parameter

$$
\hat{\delta}=\max \left\{\delta_{1}, \delta_{2}\right\}
$$

is maximized without losing quadratic stabilizability, where $\delta_{i}$ is related to the quantization density $\rho_{i}$ of quantizer $Q_{i}(\cdot), i=1,2$.

\section{A. Input and Output Sector Bound Conditions}

Consider a Lyapunov function candidate $V(\bar{x})=\bar{x}^{T} P \bar{x}$ with $P=P^{T}>0$ for the closed-loop system (8). We define

$$
\Phi=f\left(x, \xi, \delta_{1}, \delta_{2}\right)^{T} P f\left(x, \xi, \delta_{1}, \delta_{2}\right)-(1-\varepsilon) \bar{x}^{T} P \bar{x}
$$

where $\Phi:=\Phi\left(x, \xi, \delta_{1}, \delta_{2}, \varepsilon\right)$ and $\varepsilon$ is a positive scalar.

Then, along the trajectory of (8), we have

$$
V(\bar{x}(k+1))-V(\bar{x}(k))<\Phi\left(x(k), \xi(k), \delta_{1}, \delta_{2}, \varepsilon\right) .
$$

Hence, (8) is quadratically stable if and only if there exists some $P=P^{T}>0$ and $\varepsilon>0$ such that

$$
\Phi\left(x, \xi, \delta_{1}, \delta_{2}, \varepsilon\right) \leq 0, \forall x, \xi
$$

Define

$$
\begin{aligned}
& \bar{A}\left(\Delta_{1}, \Delta_{2}\right)=\left[\begin{array}{cc}
A & 0 \\
0 & A_{c}
\end{array}\right] \\
& \quad+\left[\begin{array}{c}
B\left(1+\Delta_{2}\right)\left[\begin{array}{ll}
0 & C_{c}
\end{array}\right]+D_{c}\left(1+\Delta_{1}\right)\left[\begin{array}{ll}
C & 0
\end{array}\right] \\
B_{c}\left(1+\Delta_{1}\right)\left[\begin{array}{ll}
C & 0
\end{array}\right]
\end{array}\right]
\end{aligned}
$$

and

$$
\Omega\left(\Delta_{1}, \Delta_{2}\right)=\bar{A}\left(\Delta_{1}, \Delta_{2}\right)^{T} P \bar{A}\left(\Delta_{1}, \Delta_{2}\right)-P
$$

The first result of this section is given below.

Theorem 4.1: Consider the closed-loop system (8) and some given $P=P^{T}>0$. Then, (17) holds for some $\varepsilon>0$ if and only if

$$
\Omega\left(\Delta_{1}, \Delta_{2}\right)<0, \quad \forall\left|\Delta_{1}\right| \leq \delta_{1},\left|\Delta_{2}\right| \leq \delta_{2} .
$$

The proof is given in the Appendix.

\section{B. Quadratic Stabilization}

From Theorem 4.1, it follows that the quadratic stability of the closed-loop system (1)-(3) is equivalent to the quadratic stability of the auxiliary system

$$
\left\{\begin{aligned}
x(k+1) & =A x(k)+B\left(1+\Delta_{2}\right) w(k) \\
\xi(k+1) & =A_{c} \xi(k)+B_{c}\left(1+\Delta_{1}\right) y(k) \\
y(k) & =C x(k) \\
w(k) & =C_{c} \xi(k)+D_{c}\left(1+\Delta_{1}\right) y(k)
\end{aligned}\right.
$$

where $\left|\Delta_{1}\right| \leq \delta_{1},\left|\Delta_{2}\right| \leq \delta_{2}$, and the parameter $\delta_{i}$ is related to the quantization density $\rho_{i}$ via $\delta_{i}=\left(1-\rho_{i}\right) /\left(1+\rho_{i}\right)$, $i=1,2$.

Now, define the following auxiliary notation:

$$
\begin{gathered}
\hat{A}=\left[\begin{array}{cc}
A+B D_{c} C & B C_{c} \\
B_{c} C & A_{c}
\end{array}\right], \quad \hat{B}=\left[\begin{array}{cc}
B & B D_{c} \\
0 & B_{c}
\end{array}\right], \\
\hat{C}=\left[\begin{array}{cc}
D_{c} C & C_{c} \\
C & 0
\end{array}\right], \quad \hat{D}=\left[\begin{array}{cc}
0 & D_{c} \\
0 & 0
\end{array}\right], \\
q(k)=\left[\begin{array}{ll}
q_{1} & q_{2}
\end{array}\right]^{T}, \quad p(k)=\left[\begin{array}{ll}
p_{1} & p_{2}
\end{array}\right]^{T}, \\
q_{1}=w(k), \quad q_{2}=y(k), \quad p_{1}=\Delta_{1} q_{1}, \quad p_{2}=\Delta_{2} q_{2} .
\end{gathered}
$$

Through standard linear fractional transformations [15], the closed-loop system (21) can be recast as

$$
\left\{\begin{aligned}
\bar{x}(k+1) & =\hat{A} \bar{x}+\hat{B} p(k) \\
q(k) & =\hat{C} \bar{x}+\hat{D} p(k) \\
p(k) & =\hat{\Delta} q(k), \quad \hat{\Delta}=\operatorname{diag}\left\{\Delta_{1}, \Delta_{2}\right\} .
\end{aligned}\right.
$$

Let $\hat{G}(z)$ be the transfer function matrix from $p(z)$ to $q(z)$ of the open-loop system in (23), i.e.,

$$
\hat{G}(z)=\hat{C}(z I-\hat{A})^{-1} \hat{B}+\hat{D} .
$$

Then, the closed-loop system in (23) is quadratically stable if the following small-gain condition [16] holds:

$$
\|\hat{G}(z)\|_{\infty}\|\hat{\Delta}\|_{2}<1 \text {. }
$$

It turns out that for a single uncertainty block the smallgain condition is a necessary and sufficient condition to assure the quadratic stability of system (23) (see [17]). However, for multiple uncertainty blocks the small-gain condition can be conservative to assess the quadratic stability. To avoid the conservativeness, we apply a scaled small-gain condition as follows:

$$
\left\|T \hat{G}(z) T^{-1}\right\|_{\infty}\|\hat{\Delta}\|_{2}<1
$$

where $T$ is any invertible diagonal matrix [18]. Without loss of generality, we can take $T=\operatorname{diag}\{1, \tau\}, \tau>0$.

In view of the above, we give the following result to assess the quadratic stability of the closed-loop system (1)-(3).

Theorem 4.2: Consider the system (1) and quantizers as in (3) with given densities $\rho_{1}$ and $\rho_{2}$. This system is 
quadratically stabilizable via the controller (2) if and only if the auxiliary system

$$
\left\{\begin{aligned}
x(k+1) & =A x(k)+B\left(1+\Delta_{2}\right) w(k) \\
v(k) & =\left(1+\Delta_{1}\right) C x(k),\left|\Delta_{i}\right| \leq \delta_{i}, i=1,2
\end{aligned}\right.
$$

is quadratically stabilizable via the controller (2). This in turns is guaranteed when $\delta_{i}<\hat{\delta}_{\text {sup }}, i=1,2$, where

$$
\hat{\delta}_{\text {sup }}=\frac{1}{\inf _{K, T}\left\|T \hat{G}(z) T^{-1}\right\|_{\infty}}
$$

with $\hat{G}(z)$ as given in (24), $T$ is a diagonal and invertible matrix and

$$
K=\left[\begin{array}{cc}
A_{c} & B_{c} \\
C_{c} & D_{c}
\end{array}\right] .
$$

Proof. The proof of the equivalence between the quadratic stability of the quantized and the uncertain system is straightforward from Theorem 4.1. On the other hand, the upper bound $\delta_{\text {sup }}$ for $\delta_{i}, i=1,2$ follows from the scaled small-gain condition (25) and by noting that $\|\hat{\Delta}\|_{2} \leq \max \left\{\delta_{1}, \delta_{2}\right\}$.

Remark 4.1: For a single quantizer, Theorem 4.2 becomes equivalent to Theorem 3.1, since the small-gain theorem is necessary and sufficient for quadratic stability.

Remark 4.2: The joint design of $K$ and $T$ in Theorem 4.2 leads to a non-convex $H_{\infty}$ optimization problem. Nevertheless, for a given invertible matrix $T$, the $H_{\infty}$ optimization problem is convex and the controller can be determined, for instance, via the LMI framework [19].

\section{Bandwidth Allocation}

The quantizer defined in (5) has an infinite number of quantization levels and thus it is not practical. To obtain a finite quantizer, we can truncate the logarithm quantizer, as proposed in [6]. In such case, the stability is guaranteed regionally (i.e., for some set of initial conditions) and the system trajectory converges to a small neighborhood of the origin in the sense of practical quadratic stability [6, Definition 5.3]. We emphasize that finite logarithmic quantizers need just few bits to hold a similar performance of infinite quantizers [6], [9].

In this setting, we are interested in allocating the joint quantization density assuming that the quantizers are independent and share the same communication channel. To this end, we need a cost function to represent the joint quantization density for the given logarithmic quantizers. Assuming the two quantizers having sector bounds $\delta_{1}$ and $\delta_{2}$, the cost function we choose is described by

$$
J\left(\delta_{1}, \delta_{2}\right)=1 / \delta_{1}+1 / \delta_{2}
$$

The above function has the property that if $\delta_{1}$ or $\delta_{2}$ approaches zero, $J\left(\delta_{1}, \delta_{2}\right)$ will approach infinity, since $\delta_{1}$ and $\delta_{2}$ approaching zero requires an infinite bandwidth. Thus, this cost function resembles in some way the notion of total quantization density. It is obvious that other cost functions can be used, but the method demonstrated here still applies. Hence, our optimization problem becomes

$$
\min _{K, T} J\left(\delta_{1}, \delta_{2}\right)
$$

subject to the quadratic stability of the quantized feedback system.

Let

$$
\tilde{G}(z)=T \hat{G}(z) T^{-1} W\left(\delta_{1}, \delta_{2}\right)
$$

with $W\left(\delta_{1}, \delta_{2}\right)=\operatorname{diag}\left\{\delta_{1}, \delta_{2}\right\}$. The quadratic stability of system (1)-(3) is guaranteed for given $\rho_{1}$ and $\rho_{2}$, if there exist matrices $K$ and $T$ such that

$$
\|\tilde{G}(z)\|_{\infty}<1 \text {. }
$$

In order to determine the pair $\left(\delta_{1}, \delta_{2}\right)$ that minimizes the cost function in (29), we can grid $\delta_{1}$ from 0 to some upper bound $\bar{\delta}_{1}$, which can be the maximum admissible $\delta$ for one quantizer, and compute $\delta_{2}, K$ and $T=\operatorname{diag}\{1, \tau\}, \tau>0$, such that (31) holds. The values $\delta_{1}^{*}$ and $\delta_{2}^{*}$ such that $J\left(\delta_{1}^{*}, \delta_{2}^{*}\right)$ is minimal give the best quantization densities in the sense of requiring a minimized bandwidth allocation.

To demonstrate the above method, consider the following system borrowed from [8, Example 3.1]

$$
G(z)=\frac{z-3}{z(z-2)} .
$$

The above system with one quantizer is output feedback quadratically stabilizable for $\delta_{\text {sup }}=0.1$. To allocate the joint quantization density, we grid $\delta_{1}$ from 0 to $\bar{\delta}_{1}=0.1$ and determine the scalar $\delta_{2}$ and the matrices $K$ and $T$ such that

$$
\sup _{\delta_{2}, \tau, K}\|\tilde{G}(z)\|_{\infty}=1 .
$$

The results obtained for $\delta^{*}=1 / J\left(\delta_{1}^{*}, \delta_{2}^{*}\right)$ are displayed in Fig. 3, where we have applied Remark 4.2 to solve the problem numerically. It follows from these results that the smallest joint quantization density is achieved with $\delta_{1} \cong$ $\delta_{2} \cong 0.05$ (the point that maximizes the curve in Fig. 3), that is $\rho_{1}=\rho_{2} \cong 0.9$. We emphasize that when the system is subject to only one quantizer (configuration I or II in Section III), the above procedure leads to the same result of Theorem 3.1, demonstrating that the proposed method is not conservative in the single quantizer case.

\section{CONCLusion}

This paper has extended the sector bound approach to cope with input and output quantized linear feedback control systems. The contribution of this paper is two fold. Firstly, we have shown that the problem of quadratic stabilization via quantized feedback can be addressed with no conservatism via an auxiliary uncertain system with two sector bound conditions. Secondly, we have used a scaled $H_{\infty}$ optimization approach to estimate the coarsest quantization densities that permit quadratic stabilization. Finally, we have introduced a method for allocating the communication channel bandwidth, which is demonstrated via a numerical example. 


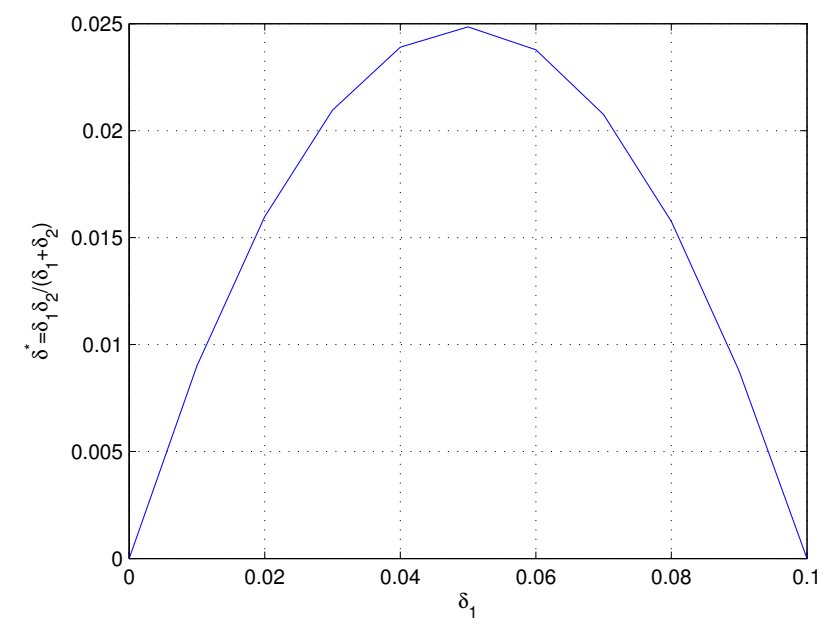

Fig. 3. Example of bandwidth allocation.

\section{APPENDIX}

\section{PROOF OF THEOREM 4.1}

First, we introduce several lemmas needed for the proof of Theorem 4.1. In the sequel, we assume that $P=P^{T}>0$.

Lemma 1.1: Suppose (17) holds. Then,

$$
\Phi\left(x, \xi, \delta_{1}-\varepsilon_{1}, \delta_{2}, \varepsilon\right) \leq 0, \quad \forall x, \xi
$$

when $\varepsilon_{1}>0$ is sufficiently small.

Proof: We first consider any $x$ such that $y=C x$ is fixed and $y \in\left[1 /\left(1+\delta_{1}\right), 1 /\left(1-\delta_{1}\right)\right)$. In this case, $Q_{1}(C x)=1$. Let

$$
g\left(y, \delta_{2}, \varepsilon\right)=\max _{x, \xi, C x=y} \Phi\left(x, \xi, \delta_{1}, \delta_{2}, \varepsilon\right) .
$$

Note that $g\left(y, \delta_{2}, \varepsilon\right)$ does not depend on $\delta_{1}$. From (8), it is clear that $g\left(y, \delta_{2}, \varepsilon\right) \leq 0$ for all $y \in\left[1 /\left(1+\delta_{1}\right), 1 /\left(1-\delta_{1}\right)\right)$.

Now consider the case that $\delta_{1}$ is reduced to $\tilde{\delta}_{1}=\delta_{1}-\varepsilon_{1}$ with $0<\varepsilon_{1}<\delta_{1}$. For $y \in\left[1 /\left(1+\tilde{\delta}_{1}\right), 1 /\left(1-\tilde{\delta}_{1}\right)\right)$, we still have $Q_{1}(y)=1$ and $g\left(y, \delta_{2}, \varepsilon\right)$ remains the same and hence $g\left(y, \delta_{2}, \varepsilon\right) \leq 0$. That is,

$$
\Phi\left(x, \xi, \tilde{\delta}_{1}, \delta_{2}, \varepsilon\right) \leq 0, \forall x, \xi: C x \in\left[1 /\left(1+\tilde{\delta}_{1}\right), 1 /\left(1-\tilde{\delta}_{1}\right)\right)
$$

Let $\tilde{\rho}_{1}=\left(1-\tilde{\delta}_{1}\right) /\left(1+\tilde{\delta}_{1}\right)$. For $y=C x \in\left[\tilde{\rho}_{1}^{i} /\left(1+\tilde{\delta}_{1}\right)\right.$, $\left.\tilde{\rho}_{1}^{i} /\left(1-\tilde{\delta}_{1}\right)\right)$, we have $Q_{1}(C x)=\tilde{\rho}_{1}^{i}$ and

$$
\Phi\left(x, \xi, \tilde{\delta}_{1}, \delta_{2}, \varepsilon\right)=\tilde{\rho}_{1}^{i} \Phi\left(\hat{x}, \hat{\xi}, \tilde{\delta}_{1}, \delta_{2}, \varepsilon\right)
$$

where $\hat{x}=x \tilde{\rho}_{1}^{-i}$ and $\hat{\xi}=\xi \tilde{\rho}_{1}^{-i}$ with $C \hat{x} \in\left[1 /\left(1+\tilde{\delta}_{1}\right), 1 /(1-\right.$ $\left.\tilde{\delta}_{1}\right)$ ). Using (34) (with $\hat{x}$ and $\hat{\xi}$ in lieu of $x$ and $\xi$ ), we get

$$
\Phi\left(x, \xi, \tilde{\delta}_{1}, \delta_{2}, \varepsilon\right) \leq 0, \forall x, \xi: C x \in\left[\tilde{\rho}_{1}^{i} /\left(1+\tilde{\delta}_{1}\right), \tilde{\rho}_{1}^{i} /\left(1-\tilde{\delta}_{1}\right)\right)
$$

for all $i$. Using the facts that $(0, \infty)$ is covered by the union of all $\left[\tilde{\rho}_{1}^{i} /\left(1+\tilde{\delta}_{1}\right), \tilde{\rho}_{1}^{i} /\left(1-\tilde{\delta}_{1}\right)\right)$ and that $\Phi\left(x, \xi, \delta_{1}, \delta_{2}, \varepsilon\right)$ is an even function of $x$, the claim in the lemma follows.

Lemma 1.2: Given a logarithmic quantizer $Q(\cdot)$ in (6) with quantization density $\rho$, let $\delta$ be given by (7) and define

$$
\Delta(v)=Q(v) / v-1, \quad v \neq 0 .
$$

Then, the following properties hold:

1) $|\Delta(v)| \leq \delta$ for any $v \neq 0$

2) For any $\Delta_{0} \in[-\delta, \delta)$, there exists a unique solution $v_{0}>0$ to $\Delta(v)=\Delta_{0}$ in $v \in[1 /(1+\delta), 1 /(1-\delta))$. Moreover, all the solutions of $v$ in $(0, \infty)$ are given by $\pm \rho^{i} v_{0}, i=0, \pm 1, \pm 2, \cdots$.

These properties are easily verified, so the proof is omitted.

Lemma 1.3 ([14]): Given any irrational number $\alpha$, there exists a sequence $\left(n_{k}, d_{k}\right), k=1,2, \ldots$, such that $n_{k}$ and $d_{k}$ are coprime, $d_{k} \rightarrow \infty$ as $k \rightarrow \infty$ and

$$
\left|\frac{n_{k}}{d_{k}}-\alpha\right| \leq \frac{1}{d_{k}}, \quad \forall k=1,2, \ldots
$$

Proof of Theorem 4.1: We first show the sufficiency. Suppose $\Omega\left(\Delta_{1}, \Delta_{2}\right)<0$ for all $\left|\Delta_{1}\right| \leq \delta_{1}$ and $\left|\Delta_{2}\right| \leq \delta_{2}$. By continuity, there exists some small $\varepsilon>0$ such that $\Omega\left(\Delta_{1}, \Delta_{2}\right)+\varepsilon P \leq 0$ for all $\left|\Delta_{1}\right| \leq \delta_{1}$ and $\left|\Delta_{2}\right| \leq \delta_{2}$. Now, a direct consequence of Lemma 1.2 is that $Q_{1}(v)=$ $\left(1+\Delta_{1}(v)\right) v$ with $\left|\Delta_{1}(v)\right| \leq \delta_{1}$ for any $v$. A similar result holds for $Q_{2}(\cdot)$. Hence, we can write

$$
\begin{aligned}
& \Phi\left(x, \xi, \delta_{1}, \delta_{2}, \varepsilon\right) \\
& \quad=\left[\begin{array}{ll}
x^{T} & \xi^{T}
\end{array}\right]\left(\Omega\left(\Delta_{1}\left(v_{1}\right), \Delta_{2}\left(v_{2}\right)\right)+\varepsilon P\right)\left[x^{T} \xi^{T}\right]^{T}
\end{aligned}
$$

with $\left|\Delta_{1}\left(v_{1}\right)\right| \leq \delta_{1}$ and $\left|\Delta_{2}\left(v_{2}\right)\right| \leq \delta_{2}$, where $v_{1}=C x$ and $v_{2}=C_{c} \xi+D_{c}\left(1+\Delta_{1}\left(v_{1}\right)\right) v_{1}$. Hence, $\Phi\left(x, \xi, \delta_{1}, \delta_{2}, \varepsilon\right) \leq 0$ for all $x, \xi$ for the chosen $\varepsilon>0$.

To prove the necessity, we assume that $\Phi\left(x, \xi, \delta_{1}, \delta_{2}, \varepsilon\right) \leq$ 0 for all $x$ and $\xi$, for some $\varepsilon>0$. The proof is done by contradiction. To this end, we assume that there exist some $\left|\Delta_{1}^{0}\right| \leq \delta_{1},\left|\Delta_{2}^{0}\right| \leq \delta_{2}$ and nonzero $\bar{x}_{0}=\left[x_{0}^{T}, \xi_{0}^{T}\right]^{T}$ such that $\bar{x}_{0}^{T} \Omega\left(\Delta_{1}^{0}, \Delta_{2}^{0}\right) \bar{x}_{0} \geq 0$. By continuity, this implies that there exist some $\left|\Delta_{1}^{0}\right|<\delta_{1}$ and $\left|\Delta_{2}^{0}\right|<\delta_{2}$ (obtained by "shrinking" the previous $\Delta_{1}^{0}$ and $\Delta_{2}^{0}$ a bit if necessary) such that

$$
\bar{x}_{0}^{T}\left(\Omega\left(\Delta_{1}^{0}, \Delta_{2}^{0}\right)+(\varepsilon / 3) P\right) \bar{x}_{0} \geq 0 .
$$

Also by continuity, in the event that $C x_{0}=0$, we may perturb $x_{0}$ slightly so that $C x_{0}$ become nonzero and (37) is relaxed to

$$
\bar{x}_{0}^{T}\left(\Omega\left(\Delta_{1}^{0}, \Delta_{2}^{0}\right)+(\varepsilon / 2) P\right) \bar{x}_{0} \geq 0 .
$$

We need to show that (38) leads to a contradiction. We first consider the case where $\ln \rho_{2} / \ln \rho_{1}$ is an irrational number. Using Lemma 1.2, we know that all the solutions to $\Delta_{1}\left(v_{1}\right)=\Delta_{1}^{0}$ are given by $\pm v_{1}^{0} \rho_{1}^{i}, i=0, \pm 1, \pm 2, \ldots$ for some $v_{1}^{0}>0$. Similarly, all the solutions to $\Delta_{2}\left(v_{2}\right)=\Delta_{2}^{0}$ are given by $\pm v_{2}^{0} \rho_{2}^{j}, j=0, \pm 1, \pm 2, \ldots$ for some $v_{2}^{0}>0$.

Define

$$
x^{(0)}=g_{0} x_{0}, \quad \xi^{(0)}=g_{0} \xi_{0}
$$

with $g_{0}=v_{1}^{0} / C x_{0}$. We have

$$
Q_{1}\left(C x^{(0)}\right)=Q_{1}\left(v_{1}^{0}\right)=\left(1+\Delta_{1}^{0}\right) C x^{(0)} .
$$

Denote

$$
w^{0}=C_{c} \xi^{(0)}+D_{c}\left(1+\Delta_{1}^{0}\right) C x^{(0)}
$$




$$
\alpha=\ln \rho_{2} / \ln \rho_{1}, \quad \beta=\ln \left(v_{2}^{0} / w^{0}\right) / \ln \rho_{1} .
$$

Using Lemma 1.3, there exists a sequence of $\left(n_{k}, d_{k}\right)$ with the properties described in Lemma 1.3. We can always choose $m_{k}$ be such that

$$
\left|\frac{m_{k}}{d_{k}}-\beta\right| \leq \frac{1}{d_{k}} .
$$

Since $n_{k}$ and $d_{k}$ are coprime, there exists a unique solution of $\left(i_{k}, j_{k}\right)$ to

$$
i_{k} d_{k}-j_{k} n_{k}=m_{k}, \quad 0 \leq j_{k}<d_{k} .
$$

Using the above, we get

$$
\begin{aligned}
& \left|i_{k}-j_{k} \alpha-\beta\right| \\
& \quad=\left|\left(i_{k}-j_{k} \frac{n_{k}}{d_{k}}-\frac{m_{k}}{d_{k}}\right)+j_{k}\left(\frac{n_{k}}{d_{k}}-\alpha\right)+\left(\frac{m_{k}}{d_{k}}-\beta\right)\right| \\
& \quad \leq d_{k} \frac{1}{d_{k}^{2}}+\frac{1}{d_{k}}=\frac{2}{d_{k}} .
\end{aligned}
$$

It follows that

$$
\left|i_{k} \ln \rho_{1}-j_{k} \ln \rho_{2}-\ln \left(v_{2}^{0} / w^{0}\right)\right| \leq \eta_{k}
$$

or, alternatively,

$$
w^{0} \rho_{1}^{i_{k}}=e^{\eta_{k}} v_{2}^{0} \rho_{2}^{j_{k}}
$$

where

$$
\left|\eta_{k}\right| \leq \frac{2 \ln \rho_{1}}{d_{k}} \rightarrow 0, \quad k \rightarrow \infty .
$$

Now considering

$$
x^{(k)}=\rho_{1}^{i_{k}} x^{(0)}, \quad \xi^{(k)}=\rho_{1}^{i_{k}} \xi^{(0)}
$$

and using the definition of $w^{0}$, we get

$$
\begin{aligned}
& Q_{2}\left(C_{c} \xi^{(k)}+D_{c} Q_{1}\left(C x^{(k)}\right)\right) \\
& \quad=Q_{2}\left(w_{0} \rho_{1}^{i_{k}}\right)=Q_{2}\left(e^{\eta_{k}} v_{2}^{0} \rho_{2}^{j_{k}}\right)=\rho_{2}^{j_{k}} Q_{2}\left(e^{\eta_{k}} v_{2}^{0}\right)
\end{aligned}
$$

Since $\left|\Delta_{2}^{0}\right|<\delta_{2}$ (a strict inequality), we must have $v_{2}^{0} \in\left(1 /\left(1+\delta_{2}\right), 1 /\left(1-\delta_{2}\right)\right)$ (an open interval). Hence, for sufficiently large $k, \eta_{k}$ will be sufficiently small, so $e^{\eta_{k}} v_{2}^{0} \in\left(1 /\left(1+\delta_{2}\right), 1 /\left(1-\delta_{2}\right)\right)$ and $Q_{2}\left(e^{\eta_{k}} v_{2}^{0}\right)=\left(1+\Delta_{2}\right) v_{2}^{0}$. Therefore,

$$
\begin{aligned}
Q_{2} & \left(C_{c} \xi^{(k)}+D_{c} Q_{1}\left(C x^{(k)}\right)\right) \\
& =\rho_{2}^{j_{k}}\left(1+\Delta_{2}^{0}\right) v_{2}^{0} \\
& =\left(1+\Delta_{2}^{0}\right) e^{-\eta_{k}} \rho_{1}^{i_{k}} w^{0} \\
& \left.=e^{-\eta_{k}}\left(1+\Delta_{2}^{0}\right)\right)\left(\left[\begin{array}{ll}
0 & C_{c}
\end{array}\right]+D_{c}\left(1+\Delta_{1}^{0}\right)\left[\begin{array}{ll}
C & 0
\end{array}\right]\right) \bar{x}^{(k)}
\end{aligned}
$$

Hence, we can write

$$
\Phi\left(x^{(k)}, \xi^{(k)}, \delta_{1}, \delta_{2}, \varepsilon\right)=\left(\bar{x}^{(k)}\right)^{T}\left(\Omega\left(\Delta_{1}^{0}, \Delta_{2}^{0}, \eta_{k}\right)+\varepsilon P\right) \bar{x}^{(k)}
$$

where

$$
\Omega\left(\Delta_{1}^{0}, \Delta_{2}^{0}, \eta_{k}\right) \rightarrow \Omega\left(\Delta_{1}^{0}, \Delta_{2}^{0}\right), \quad k \rightarrow \infty .
$$

Using $\bar{x}^{(k)}=\rho_{1}^{i_{k}} g_{0} \bar{x}_{0}$ and (38), it follows from the above that

$$
\Phi\left(x^{(k)}, \xi^{(k)}, \delta_{1}, \delta_{2}, \varepsilon\right) \geq(\varepsilon / 4)\left(\bar{x}^{(k)}\right)^{T} P x^{(k)}>0
$$

for some sufficiently large $k$. This contradicts the assumption that $\Phi\left(x, \xi, \delta_{1}, \delta_{2}, \varepsilon\right) \leq 0$ for all $x, \xi$. This contradiction implies that $\Omega\left(\Delta_{1}, \Delta_{2}\right)<0$ for all $\left|\Delta_{1}\right| \leq \delta_{1}$ and $\left|\Delta_{2}\right| \leq \delta_{2}$.

Finally, we consider the case where $\ln \rho_{2} / \ln \rho_{1}$ is a rational number. In this case, we can perturb $\delta_{1}$ slightly to give $\tilde{\delta}_{1}=\delta_{1}-\varepsilon_{1}$ for some arbitrarily small $\varepsilon_{1}$ so that $\ln \rho_{2} / \ln \tilde{\rho}_{1}$ is irrational, where $\tilde{\rho}_{1}$ is the corresponding perturbed $\rho_{1}$. Now the proof for the irrational case can apply and we have $\Omega\left(\Delta_{1}, \Delta_{2}\right)<0$ for all $\left|\Delta_{1}\right| \leq \tilde{\delta}_{1}$ and $\left|\Delta_{2}\right| \leq \delta_{2}$. Since $\tilde{\delta}_{1}$ can be made arbitrarily close to $\delta_{1}, \Omega\left(\Delta_{1}, \Delta_{2}\right)<0$ still holds for $\left|\Delta_{1}\right| \leq \delta_{1}$ and $\left|\Delta_{2}\right| \leq \delta_{2}$ by continuity.

\section{REFERENCES}

[1] R. E. Kalman, "Nonlinear aspects of sampled-data control systems," in Proc. Symp. Nonlinear Circuit Theory, vol. VII, Brooklyn NY, 1956.

[2] D. F. Delchamps, "Stabilizing a Linear System with Quantized State Feedback," IEEE Trans. Autom. Contr., vol. 35, no. 8, pp. 916-924, 1990.

[3] W. S. Wong and R. W. Brockett, "Systems with finite communication bandwidth constraints II: Stabilization with limited information feedback," IEEE Trans. Autom. Contr., vol. 44, no. 5, pp. 1049-1053, 1999.

[4] R. W. Brockett and D. Liberzon, "Quantized feedback stabilization of linear systems," IEEE Trans. Autom. Contr., vol. 45, no. 7, pp. 12791289,2000

[5] G. N. Nair and R. J. Evans, "Stabilization with data-rate-limited feedback: tightest attainable bounds," Systems and Control Letters, vol. 41, pp. 49-56, 2000.

[6] N. Elia and K. Mitter, "Stabilization of Linear Systems with Limited Information," IEEE Trans. Autom. Contr., vol. 46, no. 9, pp. 1384$1400,2001$.

[7] G. N. Nair and R. J. Evans, "Exponential stabilization of multidimensional linear systems," Automatica, vol. 39, pp. 585-593, 2003.

[8] M. Fu and L. Xie, "The sector bound approach to quantized feedback control," IEEE Trans. Autom. Contr., vol. 50, no. 11, pp. 1698-1711, 2005.

[9] M. Fu and L. Xie, "Finite-Level Quantized Feedback Control for Linear Systems," in Proc. 45th IEEE Conf. Decision Control, Dec. 2006.

[10] G. Zhai, Y. Matsumoto, X. Chen, J. Imae and T. Kobayashi, "Hybrid Stabilization of Discrete-time LTI Systems with Two Quantized Signals," Int. J. Appl. Math. Comput. Sci., vol. 15, no. 4, pp. 509-516, 2005.

[11] B. Picasso and A. Bicchi, "On the Stabilization of Linear Systems Under Assigned I/O Quantization," IEEE Trans. Autom. Contr., vol. 52 , no. 10, pp. 1994-2000, 2007

[12] A. Packard and J. C. Doyle, "Quadratic stability with real and complex perturbations," IEEE Trans. Autom. Contr., vol. 35, no. 2, pp. 198-201, 1990.

[13] C. E. de Souza, M. Fu and L. Xie, "H-infinity analysis and synthesis of discrete-time systems with time-varying uncertainty," IEEE Trans. Autom. Contr., vol. 38, no. 3, pp. 459-462, 1993.

[14] J. Butcher, "Approximation of irrational numbers by rational numbers," New Zealand Math. Mag., vol. 36, no. 2, p. 58, 1999.

[15] K. Zhou, J. C. Doyle and K. Glover, Robust and Optimal Control, Prentice Hall, 1996.

[16] B. D. O. Anderson, "The Samll-Gain Theorem, the Passivity Theorem and their Equivalence," J. Franklin Institute, vol. 293, no. 2, pp. 105$115,1972$.

[17] J. C. Doyle and G. Stein, "Multivariable Feedback Design: Concepts for a Classical/Modern Synthesis," IEEE Trans. Autom. Contr., vol. 26 , no. 1, pp. 04-16, 1981.

[18] J. S. Shamma, "Robust Stability with Time-Varying Structured Uncertainty," IEEE Trans. Autom. Contr., vol. 39, no. 4, pp. 714-724, 1994

[19] M. C. de Oliveira, J. C. Geromel and J. Bernussou "Extended $\mathrm{H}_{2}$ and $\mathrm{H}_{\infty}$ norm characterizations and controller parametrizations for discrete-time systems," Int. J. Contr., vol. 75, no. 9, pp. 666-679, 2002. 